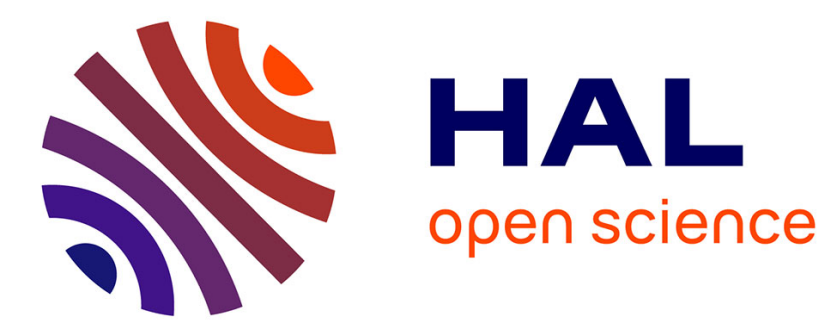

\title{
Subdomain Perturbation Finite-Element Method for Skin and Proximity Effects
}

\author{
Patrick Dular, Ruth V. Sabariego, Laurent Krähenbühl
}

\section{To cite this version:}

Patrick Dular, Ruth V. Sabariego, Laurent Krähenbühl. Subdomain Perturbation Finite-Element Method for Skin and Proximity Effects. IEEE Transactions on Magnetics, 2008, 44 (6), pp.738-741. 10.1109/TMAG.2007.915817 . hal-00287809

\section{HAL Id: hal-00287809 \\ https://hal.science/hal-00287809}

Submitted on 12 Jun 2008

HAL is a multi-disciplinary open access archive for the deposit and dissemination of scientific research documents, whether they are published or not. The documents may come from teaching and research institutions in France or abroad, or from public or private research centers.
L'archive ouverte pluridisciplinaire HAL, est destinée au dépôt et à la diffusion de documents scientifiques de niveau recherche, publiés ou non, émanant des établissements d'enseignement et de recherche français ou étrangers, des laboratoires publics ou privés. 


\title{
Subdomain Perturbation Finite-Element Method for Skin and Proximity Effects
}

\author{
Patrick Dular $^{1,2}$, Ruth V. Sabariego ${ }^{1}$, and Laurent Krähenbühl ${ }^{3}$ \\ ${ }^{1}$ Department of Electrical Engineering and Computer Science, University of Liège, ${ }^{2}$ FNRS, B-4000 Liège, Belgium \\ ${ }^{3}$ Laboratoire Ampère, Université de Lyon, Lyon, Ecole Centrale de Lyon, F-69003 Ecully Cedex, France
}

\begin{abstract}
Skin and proximity effects are calculated in both active and passive conductors via a subproblem finite-element method based on a perturbation technique. A reference limit problem considering either perfectly electric or magnetic conductors is first solved. It gives the source for eddy-current perturbation subproblems in each conductor with its actual conductivity or permeability and its own mesh. These subproblems accurately determine the current density distributions and ensuing losses in conductors of any shape in both frequency and time domains, overcoming the limitations of the impedance boundary condition technique.
\end{abstract}

Index Terms—Eddy currents, finite-element method (FEM), perturbation method, skin and proximity effects.

\section{INTRODUCTION}

$\mathbf{T}$ HE PERTURBATION of finite-element (FE) solutions provides clear advantages in repetitive analyses, like in non-destructive testing and moving systems applications [1], [2]. This technique allows to benefit from previous computations instead of starting a new complete FE solution for any variation of geometrical or physical characteristics. It also allows different problem adapted meshes and computational efficiency due to the reduced size of each subproblem.

A perturbation FE method is herein developed for accurately calculating the skin and proximity effects in conductors of any properties and shapes, in both frequency and time domains. It is a generalization and extension of the method developed in [3] and [4], applied to passive and active pure conductors, respectively. Two kinds of limit problems are defined, for both perfectly electric and magnetic conductors. They give complementary reference solutions to be used as sources in perturbation problems considering then the actual finite properties. Each problem is defined in its appropriate domain and mesh, which allows domain overlapping and distinct mesh refinements. The developments are done for the magnetic vector potential FE magnetodynamic formulation, with attention on the proper discretization of the constraints peculiar to the subdomain approach. The method is validated on a test problem and its main advantages versus the impedance boundary condition (IBC) technique [5] are pointed out.

\section{REFERENCE AND Modified EdDy-CURRENT PROBlemS}

\section{A. Reference Problem and Its Strong Formulation}

A magnetodynamic problem $p$ is defined in a domain $\Omega_{p}$, with boundary $\partial \Omega_{p}=\Gamma_{p}=\Gamma_{h, p} \cup \Gamma_{b, p}$, of the 2-D or 3-D Euclidean space. The eddy-current conducting part of $\Omega_{p}$ is denoted $\Omega_{c, p}$ and the non-conducting one $\Omega_{c, p}^{C}$, with $\Omega_{p}=\Omega_{c, p} \cup \Omega_{c, p}^{C}$. Massive conductors belong to $\Omega_{c, p}$.

Digital Object Identifier 10.1109/TMAG.2007.915817

Color versions of one or more of the figures in this paper are available online at http://ieeexplore.iee.org.
A problem, defined with subscript $p=1$, is first considered. Its equations and material relations in $\Omega_{1}$ are

$$
\begin{aligned}
& \operatorname{curl} \boldsymbol{h}_{1}=j_{1}, \quad \operatorname{curl} \boldsymbol{e}_{1}=-\partial_{t} \boldsymbol{b}_{1}, \quad \operatorname{div} \boldsymbol{b}_{1}=0 \quad(1 \mathrm{a}-\mathrm{b}-\mathrm{c}) \\
& \boldsymbol{b}_{1}=\mu_{1} \boldsymbol{h}_{1}, \quad \boldsymbol{j}_{1}=\sigma_{1} \boldsymbol{e}_{1}
\end{aligned}
$$

with boundary conditions (BCs) and interface conditions (ICs)

$$
\begin{aligned}
& \boldsymbol{n} \times\left.\boldsymbol{h}_{1}\right|_{\Gamma_{h, 1}}=0, \boldsymbol{n} \times\left.\boldsymbol{e}_{1}\right|_{\Gamma_{e, 1} \subset \Gamma_{b, 1}}=0,\left.\boldsymbol{n} \cdot \boldsymbol{b}_{1}\right|_{\Gamma_{b, 1}}=0 \quad(1 \mathrm{f}-\mathrm{g}-\mathrm{h}) \\
& {\left[\boldsymbol{n} \times \boldsymbol{h}_{1}\right]_{\gamma_{1}}=\boldsymbol{j}_{s u, 1},\left[\boldsymbol{n} \times \boldsymbol{e}_{1}\right]_{\gamma_{1}}=\boldsymbol{k}_{s u, 1},\left[\boldsymbol{n} \cdot \boldsymbol{b}_{1}\right]_{\gamma_{1}}=\boldsymbol{b}_{s u, 1}(1 \mathrm{i}-\mathrm{j}-\mathrm{k})}
\end{aligned}
$$

where $\boldsymbol{h}$ is the magnetic field, $\boldsymbol{b}$ is the magnetic flux density, $\boldsymbol{e}$ is the electric field, $\boldsymbol{j}$ is the electric current density (including source and eddy currents), $\mu$ is the magnetic permeability, $\sigma$ is the electric conductivity and $\boldsymbol{n}$ is the external unit normal to a boundary. Note that (1b) is only expressed in $\Omega_{c, 1}$, whereas it is reduced to the form (1c) in $\Omega_{c, 1}^{C}$. Also, $(1 \mathrm{~g})$ is more restrictive than (1h). The notation $[\cdot]_{\gamma}=\left.\left.\cdot\right|_{\gamma^{+-}} \cdot\right|_{\gamma^{-}}$expresses the discontinuity of a quantity through any interface $\gamma$ (of both sides $\gamma^{+}$ and $\gamma^{-}$), which is allowed to be non-zero; the associated surface fields $\boldsymbol{j}_{s u, 1}, \boldsymbol{k}_{s u, 1}$, and $\boldsymbol{b}_{s u, 1}$ are usually unknown, i.e., parts of the solution. It is intended to solve successive problems, the solutions of which being added to get the solution of a complete problem. At this first step, the solution $p=1$ is a solution as a whole, called reference or source solution.

\section{B. Modified Problem Defining Perturbations}

A modification of the problem $p=1$ due to a change of permeability or conductivity in some subregions leads to a perturbation of each field quantity. Both large and small perturbations are considered. These can result from the change of properties of existing materials [1] or from the addition of new materials in the ambient region [2], which actually also amounts to changing some material properties. The governing equations and relations in another domain $\Omega_{2}$, i.e., a modified form of $\Omega_{1}$, and the BCs and ICs, are still of the form (1) with all the involved quantities relative to the new solution and the involved boundaries relative to $\Omega_{2}$. To point out the decomposition of the new solution as a perturbation of the reference one, these quantities are written as

$$
\begin{gathered}
\boldsymbol{h}=\boldsymbol{h}_{1}+\boldsymbol{h}_{2}, \boldsymbol{b}=\boldsymbol{b}_{1}+\boldsymbol{b}_{2}, \boldsymbol{j}=\boldsymbol{j}_{1}+\boldsymbol{j}_{2}, \boldsymbol{e}=\boldsymbol{e}_{1}+\boldsymbol{e}_{2} \\
\boldsymbol{j}_{s u}=\boldsymbol{j}_{s u, 1}+\boldsymbol{j}_{s u, 2}, \boldsymbol{k}_{s u}=\boldsymbol{k}_{s u, 1}+\boldsymbol{k}_{s u, 2}, \boldsymbol{b}_{s u}=\boldsymbol{b}_{s u, 1}+\boldsymbol{b}_{s u, 2}
\end{gathered}
$$



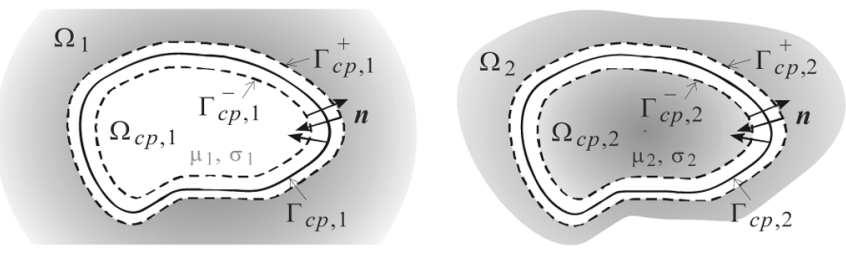

Fig. 1. Domains of the reference (left) and perturbation (right) problems ( $c p \equiv$ cpe or $c p m$ ).

Subtracting each equation of (1) from its counterpart in the so-modified problem, the perturbation equations that define the problem $p=2$, are

$$
\begin{aligned}
& \text { curl } \boldsymbol{h}_{2}=\boldsymbol{j}_{2}, \operatorname{curl} \boldsymbol{e}_{2}=-\partial_{t} \boldsymbol{b}_{2}, \operatorname{div} \boldsymbol{b}_{2}=0 \text {, } \\
& \boldsymbol{b}_{2}=\mu_{2} \boldsymbol{h}_{2}+\boldsymbol{b}_{s, 2}, \boldsymbol{j}_{2}=\sigma_{2} \boldsymbol{e}_{2}+\boldsymbol{j}_{s, 2}, \\
& \boldsymbol{n} \times\left.\boldsymbol{h}_{2}\right|_{\Gamma_{h, 2}}=0, \boldsymbol{n} \times\left.\boldsymbol{e}_{2}\right|_{\Gamma_{e, 2} \subset \Gamma_{b, 2}}=0,\left.\boldsymbol{n} \cdot \boldsymbol{b}_{2}\right|_{\Gamma_{b, 2}}=0, \quad(3 \mathrm{f}-\mathrm{g}-\mathrm{h}) \\
& {\left[\boldsymbol{n} \times \boldsymbol{h}_{2}\right]_{\gamma_{2}}=\boldsymbol{j}_{s u, 2},\left[\boldsymbol{n} \times \boldsymbol{e}_{2}\right]_{\gamma_{2}}=\boldsymbol{k}_{s u, 2},\left[\boldsymbol{n} \cdot \boldsymbol{b}_{2}\right]_{\gamma_{2}}=\boldsymbol{b}_{s u, 2} \quad(3 \mathrm{i}-\mathrm{j}-\mathrm{k})}
\end{aligned}
$$

where the so-defined volume sources $\boldsymbol{b}_{\boldsymbol{s}, 2}$ and $\boldsymbol{j}_{s, 2}$ are obtained from the reference solution as

$$
\boldsymbol{b}_{s, 2}=\left(\mu_{2}-\mu_{1}\right) \boldsymbol{h}_{1}, \quad \boldsymbol{j}_{s, 2}=\left(\sigma_{2}-\sigma_{1}\right) \boldsymbol{e}_{1}
$$

The perturbation fields are still governed by the Maxwell equations in their classical forms (3a-b-c). However, their associated material relations now include the additional sources (4a-b), that only act in the modified regions.

Solving the perturbation problem (3) instead of the modified one enables to avoid operations already performed in the reference problem (1). Moreover, at the discrete level, the meshes of both reference and perturbation problems can be simplified, with any overlapping of regions and distinct mesh refinements.

By construction, the summation of the solutions of (1) and (3), i.e., (2), gives exactly the solution of the modified problem under the condition that all materials are linear and that the wished changes in domain $\Omega_{2}$ with reference to domain $\Omega_{1}$ are expressed by (4a-b) in (3d-e).

For the sake of simplicity at the discrete level, domain $\Omega_{2}$ can disregard some materials initially present in domain $\Omega_{1}$, while these must exist in the considered complete problem. Any intersection of non-material regions of $\Omega_{2}$ with the material regions of $\Omega_{1}$ is thus allowed [2]. This means that the corrections (4a-b) are deliberately avoided in relations (3d-e) of the disregarded material regions [4]. For large perturbations, iterations between subproblems are required to obtain an accurate solution as a series of mutual perturbations. Nonlinear analyses are classically treated inside each problem, with possible inter-problem iterations.

\section{RefEREnCE PROBlem AS A Limit CASE}

\section{A. Reference Problem With Perfect Conductors}

A first reference problem considers conductors as perfect, with $\sigma_{1} \rightarrow \infty$ (see Fig. 1, left). These are denoted $\Omega_{c p e, 1} \subset$ $\Omega_{c, 1} \subset \Omega_{1}$, and their boundary $\Gamma_{c p e, 1}=\partial \Omega_{c p e, 1}$. The resulting surface currents are considered to flow between the outer and inner sides of $\Gamma_{c p e, 1}$ with regard to $\Omega_{c p e, 1}$, i.e., $\Gamma_{c p e, 1}^{+}$and $\Gamma_{c p e, 1}^{-}$. The perfect conductors $\Omega_{c p e, 1}$ are extracted from $\Omega_{1}$ in
(1) and treated via a BC of zero normal magnetic flux density on their boundaries $\Gamma_{c p e, 1}^{+}$, also occurring on $\Gamma_{c p e, 1}^{-}$, i.e.,

$$
\left.\boldsymbol{n} \cdot \boldsymbol{b}_{1}\right|_{\Gamma_{c p e, 1}^{+}}=0,\left.\quad \boldsymbol{n} \cdot \boldsymbol{b}_{1}\right|_{\Gamma_{c p e, 1}^{-}}=0 \text {. }
$$

The trace of the magnetic field is unknown on $\Gamma_{c p e, 1}^{+}$and vanishes on $\Gamma_{c p e, 1}^{-}$, i.e., with (1i)

$$
\boldsymbol{n} \times\left.\boldsymbol{h}_{1}\right|_{\Gamma_{c p e, 1}^{+}}=\boldsymbol{j}_{s u, 1}, \quad \boldsymbol{n} \times\left.\boldsymbol{h}_{1}\right|_{\Gamma_{c p e, 1}^{-}}=0 .
$$

The modified problem (3) considers the conductors $\Omega_{c p e, 2}=$ $\Omega_{c p e, 1} \subset \Omega_{c, 2}$ with their finite conductivity $\sigma_{2}$ (see Fig. 1, right). The fields in $\Omega_{c p e, 2}$ are not surface fields anymore but penetrate the conductors. They are solutions of problem (3) with $\Omega_{2}$ including $\Omega_{c p e, 2}$ with particular ICs (3i-k) through $\Gamma_{c p e, 2}=$ $\partial \Omega_{c p e, 2}$. On the one hand, (3k) with (2) and (1k) gives

$$
\left[\boldsymbol{n} \cdot \boldsymbol{b}_{2}\right]_{\Gamma_{c p e, 2}}=\boldsymbol{b}_{s u, 2}=\boldsymbol{b}_{s u^{-}} \boldsymbol{b}_{s u, 1}=[\boldsymbol{n} \cdot \boldsymbol{b}]_{\Gamma_{c p e, 2}}-\boldsymbol{b}_{s u, 1}=0
$$

due to the continuity of $\boldsymbol{n} \cdot \boldsymbol{b}$ in (2) and the zero value of $\boldsymbol{b}_{\boldsymbol{s u}, 1}$ via (6a-b). On the other hand, (3i) with (2) and (1i) gives

$$
\left[\boldsymbol{n} \times \boldsymbol{h}_{2}\right]_{\Gamma_{c p e, 2}}=\boldsymbol{j}_{s u, 2}=[\boldsymbol{n} \times \boldsymbol{h}]_{\Gamma_{c p e, 2}}-\boldsymbol{j}_{s u, 1}=-\boldsymbol{n} \times\left.\boldsymbol{h}_{1}\right|_{\Gamma_{c p e, 1}^{+}}
$$

due to the continuity of $\boldsymbol{n} \times \boldsymbol{h}$ in (2) and relation (6a).

\section{B. Reference Problem With Perfect Magnetic Materials}

Another reference problem considers conductors $\Omega_{c p m, 1} \subset$ $\Omega_{c, 1} \subset \Omega_{1}$, of boundary $\Gamma_{c p m, 1}=\partial \Omega_{c p m, 1}$, as perfect magnetic materials, i.e., with $\mu_{1} \rightarrow \infty$ (see Fig. 1, left). The domain $\Omega_{c p m, 1}$ can thus be extracted from $\Omega_{1}$ in (1) and treated via a BC fixing a zero tangential magnetic field on its boundary $\Gamma_{c p m, 1}^{+}$, also occurring on $\Gamma_{c p m, 1}^{-}$, i.e.,

$$
\boldsymbol{n} \times\left.\boldsymbol{h}_{1}\right|_{\Gamma_{c p m, 1}^{+}}=0, \quad \boldsymbol{n} \times\left.\boldsymbol{h}_{1}\right|_{\Gamma_{c p m, 1}^{-}}=0 .
$$

Also, the trace of the magnetic flux density is unknown on $\Gamma_{c p m, 1}^{+}$and vanishes on $\Gamma_{c p m, 1}^{-}$, i.e., with $(1 \mathrm{k})$

$$
\left.\boldsymbol{n} \cdot \boldsymbol{b}_{1}\right|_{\Gamma_{c p m, 1}^{+}}=\boldsymbol{b}_{s u, 1},\left.\quad \boldsymbol{n} \cdot \boldsymbol{b}_{1}\right|_{\Gamma_{c p m, 1}^{-}}=0 .
$$

The modified problem (3) considers $\Omega_{c p m, 2}=\Omega_{c p m, 1}$ with its finite permeability $\mu_{2}$ (see Fig. 1 , right). The perturbation fields are solutions of problem (3) with $\Omega_{2}$ including $\Omega_{c p m, 2}$ with particular ICs $(3 \mathrm{i}-\mathrm{k})$ through $\Gamma_{c p m, 2}=\partial \Omega_{c p m, 2}$. On the one hand, (3k) with (2) and (1k) gives

$$
\left[\boldsymbol{n} \cdot \boldsymbol{b}_{2}\right]_{\Gamma_{c p m, 2}}=\boldsymbol{b}_{s u, 2}=[\boldsymbol{n} \cdot \boldsymbol{b}]_{\Gamma_{c p m, 2}}-\boldsymbol{b}_{s u, 1}=-\left.\boldsymbol{n} \cdot \boldsymbol{b}_{1}\right|_{\Gamma_{c p m, 1}^{+}}
$$

due to the continuity of $\boldsymbol{n} \cdot \boldsymbol{b}$ in (2) and relation (10a). On the other hand, (3i) with (2) and (1i) gives

$$
\left[\boldsymbol{n} \times \boldsymbol{h}_{2}\right]_{\Gamma_{c p m, 2}}=\boldsymbol{j}_{s u, 2}=[\boldsymbol{n} \times \boldsymbol{h}]_{\Gamma_{c p m, 2}}-\boldsymbol{j}_{s u, 1}=0
$$

due to the continuity of $\boldsymbol{n} \times \boldsymbol{h}$ in (2) and $\boldsymbol{j}_{s u, 1}=0$ via (9a-b).

\section{Finite ElEMENT WEAK FoRMulations}

\section{A. B-Conform Weak Magnetodynamic Formulations}

The eddy-current problems $p$ are defined in $\Omega_{p}$ with the magnetic vector potential formulation [6], expressing the electric field $\boldsymbol{e}_{p}$ in $\Omega_{c, p}$ via a magnetic vector potential $\boldsymbol{a}_{p}$ together with the gradient of an electric scalar potential $v_{p}$, and the magnetic 
flux density $\boldsymbol{b}_{p}$ in $\Omega_{p}$ as the curl of $\boldsymbol{a}_{p}$. The $\boldsymbol{a}-v$ magnetodynamic formulation of problem $p=1$ is obtained from the weak form of the Ampère equation (1a), i.e., [6]

$$
\begin{aligned}
& \left(\mu_{1}^{-1} \operatorname{curl} \boldsymbol{a}_{1}, \operatorname{curl} \boldsymbol{a}^{\prime}\right)_{\Omega_{1}}+\left(\sigma_{1} \partial_{t} \boldsymbol{a}_{1}, \boldsymbol{a}^{\prime}\right)_{\Omega_{c, 1}}+\left(\sigma_{1} \operatorname{grad} v_{1}, \boldsymbol{a}^{\prime}\right)_{\Omega_{c, 1}} \\
& +<\boldsymbol{n} \times \boldsymbol{h}_{1}, \boldsymbol{a}^{\prime}>_{\Gamma_{h, 1}}+<\boldsymbol{n} \times \boldsymbol{h}_{1}, \boldsymbol{a}^{\prime}>_{\Gamma_{b, 1}}=0, \forall \boldsymbol{a}^{\prime} \in F_{1}^{1}\left(\Omega_{1}\right)
\end{aligned}
$$

where $F_{1}^{1}\left(\Omega_{1}\right)$ is a gauged curl-conform function space defined on $\Omega_{1}$ and containing the basis functions for $\boldsymbol{a}$ as well as for the test function $\boldsymbol{a}^{\prime}$ (at the discrete level, this space is defined by edge FEs); $(\cdot, \cdot)_{\Omega}$ and $\langle\cdot, \cdot\rangle_{\Gamma}$, respectively, denote a volume integral in $\Omega$ and a surface integral on $\Gamma$ of the product of their vector field arguments. The surface integral term on $\Gamma_{h, 1}$ accounts for natural BCs of type (1f). The term on the surface $\Gamma_{b, 1}$ with essential BCs on $\boldsymbol{n} \cdot \boldsymbol{b}_{1}$ is usually omitted because it does not locally contribute to (13). It will be shown to be the key for the post-processing of the reference solution, a part of which being $\boldsymbol{n} \times \boldsymbol{h}_{1 \mid \Gamma_{b, 1}}$

The weak formulation of the perturbation problem (3) is

$$
\begin{aligned}
& \left(\mu_{2}^{-1} \operatorname{curl} \boldsymbol{a}_{2}, \operatorname{curl} \boldsymbol{a}^{\prime}\right)_{\Omega_{2}}+\left(\sigma_{2} \partial_{t} \boldsymbol{a}_{2}, \boldsymbol{a}^{\prime}\right)_{\Omega_{c, 2} \backslash \Omega_{c p, 2}} \\
& +\left(\sigma_{2} \operatorname{grad} v_{2}, \boldsymbol{a}^{\prime}\right)_{\Omega_{c, 2} \backslash \Omega_{c p, 2}}+\left(\sigma_{2} \partial_{t} \boldsymbol{a}_{2}, \boldsymbol{a}^{\prime}\right)_{\Omega_{c p, 2}} \\
& +\left(\sigma_{2} \operatorname{grad} v_{2}, \boldsymbol{a}^{\prime}\right)_{\Omega_{c p, 2}}+<\boldsymbol{n} \times \boldsymbol{h}_{2}, \boldsymbol{a}^{\prime}>_{\Gamma_{h, 2}} \\
& +<\left[\boldsymbol{n} \times \boldsymbol{h}_{2}\right]_{\Gamma_{c p, 2}}, \boldsymbol{a}^{\prime}>_{\Gamma_{c p, 2}}=0, \forall \boldsymbol{a}^{\prime} \in F_{2}^{1}\left(\Omega_{2}\right)
\end{aligned}
$$

where $\Omega_{c p, 2}$ stands for the perturbed conducting region, i.e., $\Omega_{c p e, 2}$ or $\Omega_{c p m, 2}$. A major consequence of the $\boldsymbol{b}$-conform formulation used is that ICs (7) or (11) and (8) or (12) are to be defined, respectively, in strong and weak senses, i.e., in $F_{2}^{1}\left(\Omega_{2}\right)$ and in a surface integral term.

\section{B. Perfect Conductors Perturbed to Real Ones}

For a perfect conductor $\Omega_{c p e, 1}, \mathrm{BC}$ (5a) leads to an essential BC on the primary unknown $\boldsymbol{a}_{1}$ that can be expressed via the definition of a surface scalar potential $u_{1}$ (in general single valued, if no net magnetic flux flows in $\Omega_{c p e, 1}$ ) [7], i.e.,

$$
\left.\boldsymbol{n} \cdot \operatorname{curl} \boldsymbol{a}_{1}\right|_{\Gamma_{c p e, 1}}=0 \Leftrightarrow \boldsymbol{n} \times\left.\boldsymbol{a}_{1}\right|_{\Gamma_{c p e, 1}}=\boldsymbol{n} \times\left.\operatorname{grad} u_{1}\right|_{\Gamma_{c p e, 1}},
$$

or a floating component of $\boldsymbol{a}_{1}$ in 2-D.

The reference formulation is of the form (13) where the perfect conductors are extracted from $\Omega_{1}$ and $\Omega_{c, 1}$ and are only involved through their boundaries $\Gamma_{c p e, 1}$ (added to $\Gamma_{b, 1}$ ) with (15) strongly defined in $F_{1}^{1}\left(\Omega_{1}\right)$.

The surface integral term $\left\langle\boldsymbol{n} \times \boldsymbol{h}_{1}, \boldsymbol{a}^{\prime}>_{\Gamma_{c p e, 1}}\right.$ is non-zero only for the function grad $u^{\prime}$ [from (15)], the value of which is then the total surface current $I_{1}$ flowing in $\Gamma_{c p e, 1}$ (this can be demonstrated from the general procedure developed in [7]). It is zero for all the other local test functions (at the discrete level, for any edge not belonging to $\Gamma_{c p e, 1}$ ). This way, the circuit relation can be expressed for each conductor $\Omega_{c p e, 1}$ and the coupling with electrical circuits is possible.

For the associated perturbation formulation (14), IC (7) is strongly expressed via the continuity of the vector potential $\boldsymbol{a}_{2}$ through $\Gamma_{c p e, 2}$. IC (8) can rather only act in a weak sense via the surface integral term related to $\Gamma_{c p e, 2}^{+}$in (14). Indeed, its involved quantity $\boldsymbol{n} \times \boldsymbol{h}_{1}$ is not known in a strong sense on $\Gamma_{c p e, 2}^{+}$, but rather in a weak sense. One has

$$
\begin{aligned}
& <\left[\boldsymbol{n} \times \boldsymbol{h}_{2}\right]_{\Gamma_{c p e, 2}}, \boldsymbol{a}^{\prime}>_{\Gamma_{c p e, 2}}=<-\boldsymbol{n} \times \boldsymbol{h}_{1}, \boldsymbol{a}^{\prime}>_{\Gamma_{c p e, 2}^{+}} \\
& =<-\boldsymbol{n} \times \boldsymbol{h}_{1}, \boldsymbol{a}^{\prime}>_{\Gamma_{c p e, 1}^{+}}=\left(\mu_{1}^{-1} \operatorname{curl} \boldsymbol{a}_{1}, \operatorname{curl} \boldsymbol{a}^{\prime}\right)_{\Omega_{1} \backslash \Omega_{c p e, 1}}
\end{aligned}
$$

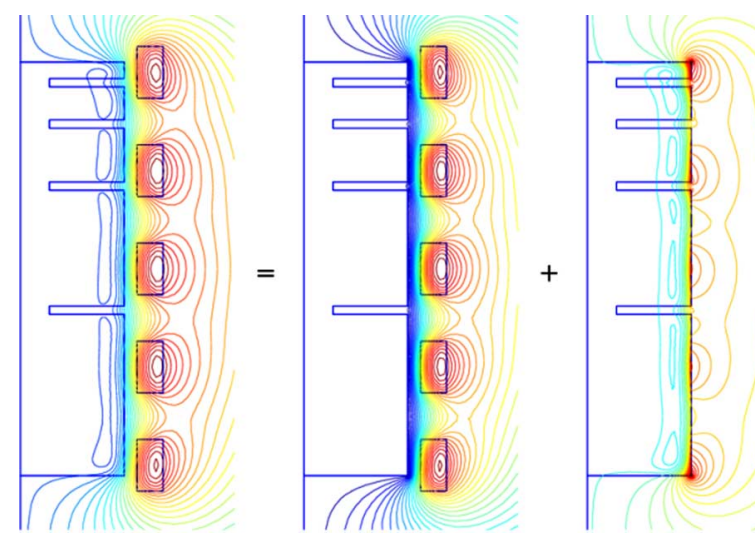

Fig. 2. Magnetic flux lines for the conventional FE solution $\boldsymbol{b}$ (left), the reference solution $\boldsymbol{b}_{1}$ (middle) and the perturbation solution $\boldsymbol{b}_{2}$ (right); system 1 with a conductive non-magnetic core.
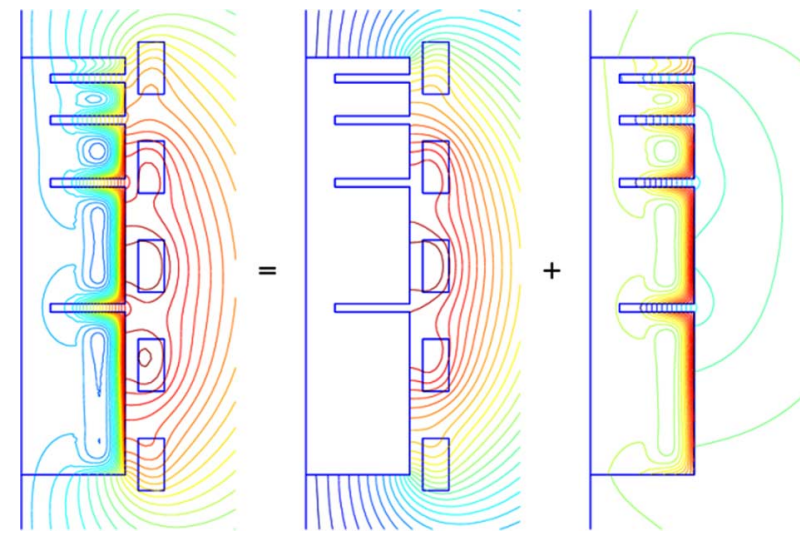

Fig. 3. Magnetic flux lines for $\boldsymbol{b}$ (left), $\boldsymbol{b}_{1}$ (middle), and $\boldsymbol{b}_{2}$ (right); system 2 with a conductive magnetic core.

in case no part of $\Omega_{c, 1} \backslash \Omega_{c p e, 1}$ is in contact with $\Omega_{c p e, 1}$ [otherwise the second and third terms of (13) have to be considered as well]. This way, the surface integral term related to $\Gamma_{c p e, 2}^{+}$in (14) is naturally calculated from a volume integral coming from the reference problem (limited at the discrete level to one single layer of FEs touching the boundary).

The discrete quantity $\boldsymbol{a}_{1}$ in (16) is initially given in the mesh of the reference problem. It has afterward to be expressed in the mesh of the perturbation problem, through a projection method [8] of its curl limited to the layer of FEs touching $\Omega_{c p e, 1}$.

\section{Perfect Magnetic Materials Perturbed to Real Ones}

When perfect magnetic materials $\Omega_{c p m, 1} \subset \Omega_{c, 1} \subset \Omega_{1}$ are considered, the reference formulation is of the form (13) where the perfect materials are extracted from $\Omega_{1}$ and $\Omega_{c, 1}$ and are only involved through their boundaries $\Gamma_{c p m, 1}$ added to $\Gamma_{h, 1}$ with the BC (9a), i.e.,

$$
<\boldsymbol{n} \times \boldsymbol{h}_{1}, \boldsymbol{a}^{\prime}>_{\Gamma_{c p m, 1} \subset \Gamma_{h, 1}}=<0, \boldsymbol{a}^{\prime}>_{\Gamma_{c p m, 1}^{+}}=0 .
$$

For the associated perturbation formulation (14), IC (11) must be strongly expressed via the vector potential $\boldsymbol{a}_{2}$. It can be expressed with a known discontinuous component $\boldsymbol{b}_{d, 2}$ of $\boldsymbol{b}_{2}$ only acting outside $\Omega_{c p m, 2}$, with $\boldsymbol{b}_{2}=\boldsymbol{b}_{c, 2}+\boldsymbol{b}_{d, 2}$, i.e.,

$$
\left[\boldsymbol{n} \cdot \boldsymbol{b}_{2}\right]_{\Gamma_{c p m, 2}}=\left.\boldsymbol{n} \cdot \boldsymbol{b}_{d, 2}\right|_{\Gamma_{c p m, 2}^{+}}=-\left.\boldsymbol{n} \cdot \boldsymbol{b}_{1}\right|_{\Gamma_{c p m, 1}^{+}}
$$



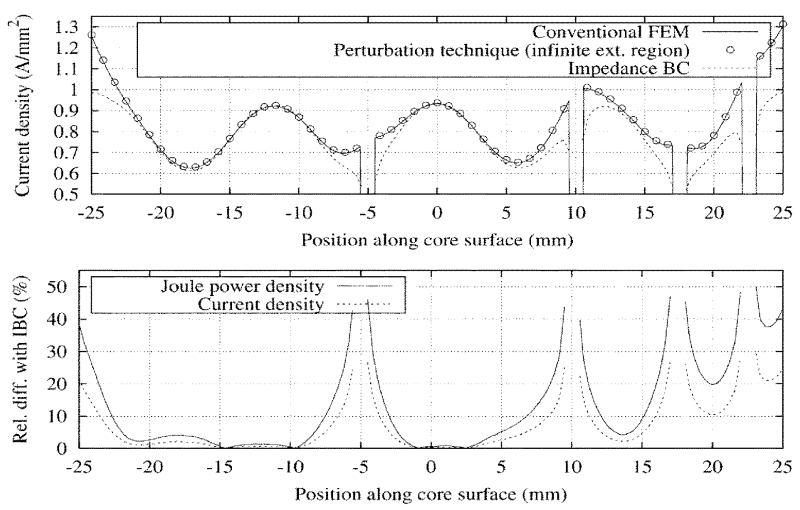

Fig. 4. Eddy-current density along the core surface for the conventional FE solution, the perturbation technique and the IBC technique (top); relative difference between solutions of the last two techniques (bottom); system 1 with a conductive non-magnetic core.
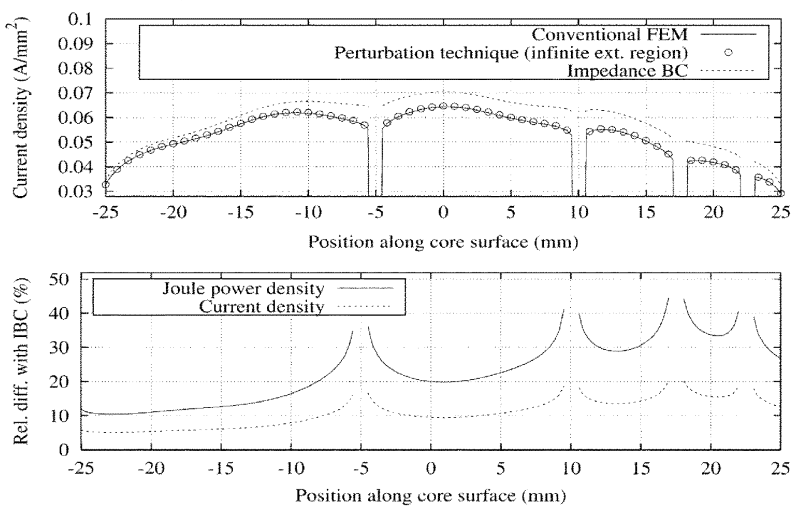

Fig. 5. Same as Fig. 4 but for system 2 with a conductive magnetic core.

or with an associated known discontinuous component $\boldsymbol{a}_{d, 2}$ of $\boldsymbol{a}_{2}$, with $\boldsymbol{a}_{2}=\boldsymbol{a}_{c, 2}+\boldsymbol{a}_{d, 2}$, also only acting outside $\Omega_{c p m, 2}$, i.e.,

$$
\left.\boldsymbol{a}_{d, 2}\right|_{\Gamma_{c p m, 2}^{+}}=-\left.\boldsymbol{a}_{1}\right|_{\Gamma_{c p m, 1}^{+}} .
$$

IC (12) cancels the surface integral term related to $\Gamma_{c p m, 2}^{+}$in (14), i.e., $<\left[\boldsymbol{n} \times h_{2}\right]_{\Gamma_{c p m, 2}}, \boldsymbol{a}^{\prime}>_{\Gamma_{c p m, 2}^{+}}=<0, \boldsymbol{a}^{\prime}>_{\Gamma_{c p m, 2}^{+}}$.

\section{APPLICATION}

Two core-inductor systems are considered as test problems (see Figs. 2 and 3). Their five copper stranded inductors are connected in series. The core of system 1 (see Fig. 2) is made of aluminium $\left(\sigma_{\mathrm{Al}}=2.710^{7} \Omega^{-1} \mathrm{~m}^{-1}\right)$. The one of system 2 (see Fig. 3$)$ is made of steel $\left(\sigma_{\mathrm{St}}=10^{6} \Omega^{-1} \mathrm{~m}^{-1}\right.$ and relative permeability $\left.\mu_{r, S t}=270\right)$. A $2-\mathrm{D}$ model with a vertical symmetry axis is considered. For a direct comparison with the IBC technique, a frequency domain analysis is done. However, the subdomain perturbation technique can be directly applied to time domain analyses without any change. The working frequencies are $5 \mathrm{kHz}$ and $500 \mathrm{~Hz}$ for systems 1 and 2, respectively, to give equal skin depths $\delta=\delta_{\mathrm{Al}}=\delta_{\mathrm{St}}=1.37 \mathrm{~mm}$. The core half-width is $12.5 \mathrm{~mm}(9.1 \delta)$ and its height is $50 \mathrm{~mm}(36.5 \delta)$.

Holes are considered in the core in order to point out the effect of several corners. They are non-uniformly distributed to allow for different lengths of plane portions between them. Small lengths should penalize the IBC technique, which is generally based on analytical solutions in practice only valid far from any geometrical discontinuities, e.g., edges and corners.

The magnetic flux lines are shown in Figs. 2 and 3 for the different calculations performed. Figs. 4 and 5 show the eddy cur- rent and Joule power density distributions in the core, as well as the relative error on these quantities made by the IBC versus the subdomain FE approach, the results of which are checked to be very similar to those of the conventional FE approach. The error significantly increases in the vicinity of the conductor corners: it reaches $50 \%$ for the Joule power density and $30 \%$ for the current density in the smallest plane portions. This affects the total losses accuracy when the size of the conductor portions decreases. The error with the IBC is shown to be significant up to a distance of about $3 \delta$ from each corner. A good accuracy is only obtained beyond this distance for system 1 . The IBC error increases with $\delta$ with respect to the structure dimensions, whereas the developed approach successfully and accurately adapts its solution to any $\delta$.

\section{CONCLUSION}

The developed subdomain perturbation FE method allows to split eddy-current analyses into subproblems of lower complexity regarding meshing operations and computational aspects. Reference solutions, related to two limit behaviors of conductors, can be used in several subproblems. This allows efficient parameterized analyses on the electric and magnetic characteristics of the conductors in a wide range, i.e., on all the parameters affecting the skin depth. Nonlinear analyses, e.g., with temperature dependent conductivities, could then benefit from this. The method is valid for conductors of any shape in both frequency and time domains, which overcomes the limitations of the IBC technique.

\section{ACKNOWLEDGMENT}

This work was supported in part by the Belgian Science Policy (IAP P6/21) and the Belgian French Community (Research Concerted Action ARC 03/08-298).

\section{REFERENCES}

[1] Z. Badics et al., "An effective 3-D finite element scheme for computing electromagnetic field distortions due to defects in eddy-current nondestructive evaluation," IEEE Trans. Magn., vol. 33, no. 2, pp. 1012-1020, Mar. 1997

[2] P. Dular and R. V. Sabariego, "A perturbation method for computing field distortions due to conductive regions with h-conform magnetodynamic finite element formulations," IEEE Trans. Magn., vol. 43, no. 4, pp. 1293-1296, Apr. 2007.

[3] P. Dular, R. V. Sabariego, J. Gyselinck, and L. Krähenbühl, "Sub-domain finite element method for efficiently considering strong skin and proximity effects," COMPEL, vol. 26, no. 4, pp. 974-985, 2007.

[4] P. Dular, R. V. Sabariego, and L. Krähenbühl, "Subdomain perturbation finite element method for strong skin and proximity effects in inductors," COMPEL, vol. 27, no. 1, pp. 72-84, 2008.

[5] L. Krähenbühl and D. Muller, "Thin layers in electrical engineering. Example of shell models in analyzing eddy-currents by boundary and finite element methods," IEEE Trans. Magn., vol. 29, no. 2, pp. 1450-1455, Mar. 1993.

[6] P. Dular, P. Kuo-Peng, C. Geuzaine, N. Sadowski, and J. P. A. Bastos, "Dual magnetodynamic formulations and their source fields associated with massive and stranded inductors," IEEE Trans. Magn., vol. 36, no. 4, pp. 3078-3081, Jul. 2000.

[7] P. Dular, J. Gyselinck, T. Henneron, and F. Piriou, "Dual finite element formulations for lumped reluctances coupling," IEEE Trans. Magn., vol. 41, no. 5, pp. 1396-1399, May 2005.

[8] C. Geuzaine, B. Meys, F. Henrotte, P. Dular, and W. Legros, "A galerkin projection method for mixed finite elements," IEEE Trans. Magn., vol. 35, no. 3, pp. 1438-1441, May 1999.

Manuscript received June 24, 2007. Corresponding author: P. Dular (e-mail: patrick.dular@ulg.ac.be). 